\title{
Determinando a ordem das cadeias de Markov usadas na modelagem do mercado de ações
}

\author{
André G.C. Pereira ${ }^{1}$ \\ Departamento de Matemática, UFRN, Natal, RN \\ Israel Smith ${ }^{2}$ \\ UFRN, Natal, RN \\ Jaques S. Lopes ${ }^{3}$ \\ Departamento de Matemática, UFRN, Natal, RN \\ Viviane S.M. Campos ${ }^{4}$ \\ Departamento de Matemática, UFRN, Natal, RN
}

\begin{abstract}
Resumo. Em muitos trabalhos sobre aplicação de cadeias de Markov no mercado de ações, a modelagem é feita via cadeias de primeira ordem. Muitos critérios de Informação (AIC, BIC, EDC, etc) foram desenvolvidos e dentre suas diversas aplicações uma delas é: Dado um conjunto de dados provenientes de uma cadeia de Markov, qual a ordem da cadeia de Markov que se ajusta melhor a esses dados? Os trabalhos analisados não se utilizaram de nenhum critério de seleção na determinação da ordem das cadeias utilizadas, e esse trabalho visa completar essa lacuna no estudo da aplicação das cadeias de Markov no mercado de ações.
\end{abstract}

Palavras-chave. Processos Estocásticos, Cadeias de Markov, Critérios de Informação.

\section{Introdução}

Em [2] é dito que as bolsas de valores são as instituições onde compra-se e/ou vende-se ações de forma sistematizada e que o único objetivo dos compradores de ações é obter lucro pelo aumento do valor das ações, entretanto existem vários diferentes motivos que podem afetar o desempenho das ações como: A tendência dos negócios à nível mundial, calamidades naturais, situação políticaeconômica, governança corporativa fraca, mudança nas políticas do governo, etc. [3] complementa que tais fatores induzem volatilidade aos preços. Em [4] acrescenta-se que a volatilidade de longo prazo está relacionada à fundamentos macroeconômicos associados com o fluxo de moeda e dos juros, enquanto a volatilidade de curto prazo é relativa a determinantes transitórios, tais como o sentimento do investidor.

Existem, então, vários fatores, internos/externos, público/governamental, de curto e longo prazo que podem causar mudança nos preços das ações de maneiras distintas, mesmo quando condições semelhantes estavam presentes antes das oscilações. Isso nos revela que o comportamento do preço das ações não é determinado de forma determinística, portanto a teoria de probabilidade tem que ser utilizada para o estudo deste fenômeno. Existem vários métodos estatísticos/probabilísticos que podem ser utilizados para estudar esse fenômeno de prever o preço

\footnotetext{
1 andre@ccet.ufrn.br

2 israelsmith2014@gmail.com

3 jaques@ccet.ufrn.br

${ }^{4}$ viviane@ccet.ufrn.br
} 
das ações usando informações passadas, como: Médias Móveis, Análise de Regressão, Modelos de Markov, Modelos de Markov Oculto, cadeias de Markov com pesos, etc. ( [2]).

Um dos métodos probabilísticos que vem sendo largamente usado para prever o movimento do preço das ações é o das cadeias de Markov, conforme podemos ver em $[2,3,5,8,11,14]$.

A propriedade advinda dos processos Markovianos, indica que um determinado processo (estocástico) tem uma memória finita, ou alcance finito, e a determinação da ordem da cadeia de Markov explicita a magnitude desse alcance. Isto é, o quanto uma dada informação, num dado instante, depende de informações anteriores do processo. Assim, se um conjunto de dados é proveniente de uma cadeia de Markov, é fundamental a determinação da ordem desta dependência que forneça o melhor ajuste.

Fato comum nos trabalhos que analisamos é que, embora existam testes para indicar a ordem da cadeia que melhor se ajusta aos dados, nenhum deles procedeu qualquer teste.

Neste artigo usamos o preço de fechamento diário de quatro ações como nossa amostra e ilustramos como utilizar os testes (o Critério de Informação de Akaike (IC),o Critério de Informação Bayesiana (BIC) e o Critério de Determinação Eficiente (EDC)) na estimação da ordem da cadeia de Markov. Uma vez determinada a ordem da cadeia de Markov procedemos a análise de convergência da mesma a fim de encontrar a sua distribuição de equilíbrio. Este artigo está dividido em quatro seções. Na Seção 2 definimos cadeias de Markov, mostramos como encontrar a matriz de transição e como verificar se a cadeia possui distribuição de equilíbrio.Na Seção 3 apresentamos os testes mais conhecidos para estimação da ordem de uma cadeia de Markov, a saber: AIC e BIC, e aproveitamos também para apresentar um teste mais robusto que os dois primeiros, o EDC. Na Seção 4 utilizamos os dados obtidos da B3 (bolsa de valores de São Paulo) para aplicar a teoria desenvolvida nas seções anteriores.

\section{Matriz de transição e distribuição de equilíbrio}

Nesta seção consideramos que a ordem da cadeia já foi determinada e usamos tal ordem para determinarmos a matriz de transição da cadeia, bem como a utilizamos para efetuar a análise da convergência. Na hipótese de convergência é determinada a distribuição de equílibrio.

Para analisarmos se uma cadeia de Markov converge, precisamos relembrar algumas definições e teoremas, cujas demonstrações podem ser encontradas em [9].

Um processo estocástico é uma sequência de variáveis aleatórias $\left\{X_{n}\right\}_{n \in \mathbb{N}}$ definidas num mesmo espaço de probabilidade $(\Omega, \mathcal{F}, P)$, onde $\Omega$ é o domínio das variáveis aleatórias $X_{n}: \Omega \rightarrow \mathbb{R}, \mathcal{F}$ é a $\sigma$-álgebra de subconjuntos de $\Omega$ e $P$ é uma probabilidade definida em $\mathcal{F}$. $S=\left\{X_{n}(w) / w \in\right.$ $\Omega$ e $n \in \mathbb{N}\}$, o conjunto dos valores assumidos por esse processo, é dito espaço de estados.

Definição $1 \mathrm{Um}$ processo estocástico $\left\{X_{n}\right\}_{n \in \mathbb{N}}$ é uma cadeia de Markov de ordem $k \in \mathbb{N}$ se satisfaz a propriedade de Markov:

$$
P\left(X_{n}=i_{n} \mid X_{0}=i_{0}, \ldots, X_{n-1}=i_{n-1}\right)=P\left(X_{n}=i_{n} \mid X_{n-1}=i_{n-1}, X_{n-2}=i_{n-2}, \ldots, X_{n-k}=i_{n-k}\right),
$$

$\forall n \in \mathbb{N}$ com $n>k$ e $i_{0}, i_{1}, \ldots, i_{n} \in S$, ou seja, o presente do processo, dada toda a sua história, é condicionalmente dependente do passado através das $k$ últimas informações. Equivalentemente, numa cadeia de Markov de ordem $k$, a próxima etapa é condicionalmente dependente das $k$ últimas etapas.

Se $\left\{X_{n}\right\}_{n \in \mathbb{N}}$ é uma cadeia de Markov de ordem 1 com espaço de estados $S$. Dizemos que:

$$
P_{i j}^{(n-1, n)}=P\left(X_{n}=j \mid X_{n-1}=i\right), \text { para } i, j \in S
$$

é a probabilidade da cadeia sair no tempo $n-1$ do estado $i$ e ir para o estado $j$ no tempo $n$. Chamamos essa probabilidade de probabilidade de transição da cadeia de Markov, que pode ou 
não depender do tempo $n$. Quando essa probabilidade independe do tempo em que o passo é dado, dizemos que a cadeia é Homogênea e escrevemos:

$$
P_{i j}=P\left(X_{n}=j \mid X_{n-1}=i\right)=P\left(X_{n+k}=j \mid X_{n+k-1}=i\right)
$$

para $k=-(n-1),-(n-2), \ldots,-1,0,1,2, \ldots$.

Seja $\left\{X_{n}\right\}_{n \in \mathbb{N}}$ uma cadeia de Markov homogênea com espaço de estados finito $S=\{1,2,3, \cdots, m\}$. Para esta cadeia existem $m^{2}$ probabilidades de transição as quais organizamos na forma de uma matriz $\mathrm{P}$, que chamamos de matriz de transição da cadeia de Markov.

$$
P=\left[\begin{array}{cccc}
P_{11} & P_{12} & \cdots & P_{1 m} \\
P_{21} & P_{22} & \cdots & P_{2 m} \\
\vdots & \vdots & \ddots & \vdots \\
P_{m 1} & P_{m 2} & \cdots & P_{m m}
\end{array}\right]
$$

Definição 2 Seja $\left\{X_{n}\right\}_{n \in \mathbb{N}}$ uma cadeia de Markov, dizemos que do estado $i$ a cadeia atinge o estado $j$ se existe $m \in \mathbb{N}$ tal que $P\left(X_{m}=j \mid X_{0}=i\right)>0$. Representamos essa situação por $i \rightarrow j$. Dizemos que a cadeia é irredutível se quaisquer que sejam $i, j \in S$ temos que $i \rightarrow j$ e $j \rightarrow i$.

Definição 3 Seja $\left\{X_{n}\right\}_{n \in \mathbb{N}}$ uma cadeia de Markov, definimos o período do estado $i$ como sendo $d(i)=m d c\left\{n \in \mathbb{N}, n \geq 1 / P_{i i}^{n}>0\right\}$. Se o período de todos os estados da cadeia for 1 , dizemos que a cadeia é aperiódica.

Teorema 1 Toda cadeia de Markov homogênea irredutível, aperiódica com espaço de estados finito é convergente, ou seja, existe $\lim _{n \rightarrow \infty} P_{i j}^{n}=\pi_{j}$ onde $\pi=\left(\pi_{1}, \ldots, \pi_{N}\right)$ é a distribuição de equilíbrio.

\section{AIC, BIC e EDC}

Para uma amostra $X_{1}, X_{2}, \ldots, X_{n}$, seja $L(\theta)$ denotando a função de verossimilhança parametrizada por $\theta$ e seja $d\left(\theta^{*}: \theta\right)=E\left\{\log \frac{L\left(\theta^{*}\right)}{L(\theta)}\right\}$ a medida de informação de Kullback-Leibler quando $\theta$ é usada para aproximar o valor verdadeiro $\theta^{*}$. O estimador de máxima verossimilhança $\widehat{\theta}$, a aproximação de $d\left(\theta^{*}: \theta\right)$ pela estatística de Neyman-Pearson junto com distribuição assintótica $\chi^{2}$ da razão de máxima verossimilhança $-2 \log \frac{L\left(\theta^{*}\right)}{L(\theta)}$, formam a base para formulação do Critério de Informação de Akaike ( [1]).

Deseja-se fazer a seleção de um modelo, onde para as hipóteses aninhadas $H_{0} \subset H_{1} \subset \cdots \subset H_{R}$, $R<\infty, H_{s}$ representa a hipótese que o processo $X=\left\{X_{n}\right\}_{n \geq 1}$ é uma Cadeia de Markov de ordem $s$. Desta maneira, se o processo $X$ assume valores em um espaço finito $S=\{1, \ldots, N\}$, [13] propôs usar AIC para selecionar a ordem e estimar a ordem verdadeira $r$ da cadeia.

Seja $X=\left\{X_{n}\right\}_{n \geq 1}$ uma Cadeia de Markov de ordem desconhecida, mas finita, $r$, com $0 \leq r \leq R<\infty$. Assumimos que $X$ toma seus valores num espaço de estados finito $S=\{1, \ldots, N\}$ e seja $P=\left(p_{i_{1} \ldots i_{r}: i_{r+1}}\right)$ denotando suas probabilidades de transição,

$$
P\left(X_{n}=i_{n} / X_{k}=i_{k}, k<n\right)=p_{i_{n-r} \ldots i_{n-1}: i_{n}} .
$$

Se $r=0$ pode ser interpretada em (1) como $p_{i_{n}}=p_{i_{n}: i_{n}}$.

Para $1 \leq s \leq R$ sejam as transições denotadas por

$$
n_{i_{1} i_{2} \ldots i_{s}}=\sum_{k=1}^{n-s+1} I_{\left(X_{k}=i_{1}, X_{k+1}=i_{2}, \ldots, X_{k+s-1}=i_{s}\right)} .
$$


e escrevamos a máxima log-verossimilhança $L_{\max }=\widehat{L}$ como

$$
\log \widehat{L}(s)=\sum_{i_{1}, \ldots, i_{s}, i_{s+1}} n_{i_{1} \ldots i_{s} i_{s+1}} \log \frac{n_{i_{1} \ldots i_{s} i_{s+1}}}{n_{i_{1} \ldots i_{s}}} .
$$

Se $n_{i_{1} \ldots i_{s} i_{s+1}}=0$, assumimos $\log \frac{n_{i_{1} \ldots i_{s} i_{s+1}}}{n_{i_{1} \ldots i_{s}}}=0$.

Para testar a hipótese de que a cadeia tem a ordem máxima $s$ contra a hipótese de que a cadeia tenha ordem máxima $r$ :

$$
\begin{gathered}
H_{s}: p_{i_{1} \ldots i_{s}: i_{s+1}} \neq p_{i_{1}^{\prime} \ldots i_{s}^{\prime}: i_{s+1}} \text { para algum }\left(i_{1}^{\prime}, \ldots, i_{s}^{\prime}\right) \neq\left(i_{1}, \ldots, i_{s}\right) \text {, o critério AIC é dado por } \\
A I C(s)=-2 \log \widehat{L}(s)+2 N^{s}(N-1),
\end{gathered}
$$

onde $N=|S|$. A ordem verdadeira $r$ pode então ser estimada por

$$
\hat{r}_{A I C}=\arg \min _{0 \leq s \leq R} A I C(s) .
$$

O termo de penalidade $2 N^{s}(N-1)$ é o número de graus de liberdade da distribuição limite $\chi^{2}$ da razão de máxima log-verossimilhança. [10] mostrou a inconsistência do estimador $\hat{r}_{A I C}$.

O Critério de Informação Bayesiana (BIC) foi proposto por [12] como uma altenativa ao AIC,

$$
B I C(s)=-2 \log \widehat{L}(s)+N^{s}(N-1) \log n,
$$

onde $n$ é o tamanho da amostra. O estimador correspondente

$$
\hat{r}_{B I C}=\arg \min _{0 \leq s \leq R} B I C(s) .
$$

é provado ser um estimador fortemente consistente, $\hat{r}_{B I C} \stackrel{a . s}{\longrightarrow} r$, quando o processo de Markov derivado de ordem simples $Y_{n}^{(r)}=\left(X_{n}, \ldots, X_{n+r-1}\right)$ é irredutível. Posteriormente, um grande avanço foi dado por [6], que estabeleceram a consistência forte para BIC sem assumir a limitação para a ordem verdadeira $r$.

Um critério mais geral, que engloba os critérios AIC e o BIC, é o Critério de Determinação Eficiente (EDC), introduzido por [15],

$$
E D C(s)=-2 \log \widehat{L}(s)+\gamma(s) c_{n}
$$

com

$$
\hat{r}_{E D C}=\arg \min _{0 \leq s \leq R} E D C(s) .
$$

onde o termo de penalidade $\gamma(s) c_{n}$ é composto por uma função positiva e estritamente crescente $\gamma(\cdot)$ e uma sequência de contantes $c_{n}$ satisfazendo

$$
\frac{c_{n}}{n} \stackrel{a . s}{\longrightarrow} 0 \quad e \frac{c_{n}}{\log \log n} \stackrel{a . s}{\longrightarrow} \infty .
$$

Mais tarde, [7] propôs um estimador consistente ótimo, baseado no critério:

$$
E D C_{\text {opt }}(s)=-2 \log \widehat{L}(s)+2|S|^{s+1} \log \log (n),
$$

onde a escolha deste termo de penalidade torna este estimador consistente melhor quando comparado ao BIC no que se refere a velocidade de convergência para a ordem verdadeira. 


\subsection{Como estimar as matrizes de transição}

Para fixarmos as notações, vamos supor que no experimento em estudo o espaço de estados seja $S=\{1,2, \ldots, N\}$ e que $X_{1}, X_{2}, \ldots, X_{n}$ seja a amostra.

O estimador escolhido fornece a ordem da cadeia de Markov que melhor se ajusta aos dados. Por exemplo, se o estimador utilizado foi o BIC e ele indicou ordem 0 isso significa que o próximo estado é escolhido de forma independente do estado atual, e com probabilidade igual a proporção entre o número de vezes que o próximo estado apareceu na amostra e o tamanho da amostra, ou seja,

$$
\hat{P}\left(X_{n+1}=j \mid X_{n}=i\right)=\hat{P}\left(X_{n+1}=j\right)=\frac{n_{j}}{n},
$$

onde $n_{j}$ é o número de vezes que o estado $j$ apareceu na amostra.

Se a ordem apontada pelo estimador for 1, a matriz de transição é construída conforme os artigos citados anteriomente, a saber:

$$
\hat{P}_{i j}= \begin{cases}\frac{n_{i j}}{n_{i}}, & \text { se } n_{i .} \neq 0 \\ \delta_{i}(j), & \text { se } n_{i .}=0\end{cases}
$$

onde $n_{i j}$ é o número de vezes que a sequência $i j$ aparece na amostra e $n_{i}$. é o número de vezes que a sequência $i k$ aparece na amostra qualquer que seja o $k \in S$.

Quando a ordem for 2 devemos lembrar que o próximo estado depende dos 2 estados anteriores. Então seria conveniente olharmos para cada estado agora como um par, ou seja, algo do tipo $i j$.É como se no tempo $n$ a cadeia estivesse no estado $i$ e no tempo $n+1$ a cadeia estivesse no estado $j$. Se no próximo tempo $n+1$ estivermos em $k p$, significa que no tempo $n+1$ a cadeia está no estado $k$ e no tempo $n+2$ está no estado $p$. Portanto, em tempos consecutivos, a cadeia ir de $i j$ para $k p$ só pode acontecer com probabilidade positiva se $j=k$, e portanto:

$$
\hat{P}_{i j, k p}= \begin{cases}0 & \text { se } j \neq k \\ \frac{n_{i j p}}{n_{i} j .} & \text { se } j=k \text { e } n_{i j .} \neq 0 \\ \delta_{i j}(k p) & \text { se } n_{i j .}=0\end{cases}
$$

onde $n_{i j p}$ é o número de vezes que a sequência $i j p$ aparece na amostra, $n_{i j}$. é o número de vezes que a sequência $i j k$, para qualquer $k \in S$, aparece na amostra e $\delta_{i j}(k p)$ é a função característica que resulta 1 se $i j=k p$ e em 0 caso contrário.

E o processo segue de maneira análoga qualquer que seja a ordem indicada pelo estimador.

Quando a ordem for zero não temos uma cadeia de Markov, visto que o próximo passo não depende do anterior, o processo já se move como se estivesse em equilíbrio e a distribuição de equilíbrio é a própria proporção calculada no início.

Nos outros casos temos uma matriz de transição e o procedimento para analisarmos a convergênica é sempre o mesmo: devemos verificar se a cadeia é irredutível, aperiódica e finita. Se essas condições forem satisfeitas, então teremos uma distribuição de equilíbrio conforme o Teorema 1.

\section{Exemplos numéricos}

Nesta seção usamos os preços de fechamento das ações GOLL4,CIEL3,JBSS3,ABEV3 obtidos do site yahoo finance de todo o ano de 2018, num total de 245 amostras para cada ação. Da mesma forma que é feito nas referências citadas, usamos os preços para determinar a variação diária do preço da ação, obtendo a sequência dos retornos diários. Assim, se $P_{n}$ é o preço da ação no $n$-ésimo dia, o seu rendimento nesse dia foi $R_{n}=\left(P_{n}-P_{n-1}\right) / P_{n-1}$ e é esse rendimento que vai ser modelado pela cadeia de Markov. Neste trabalho, para discretizar o modelo, dividimos os 
rendimentos em faixas e cada faixa recebe um número, assim usando a sequência $R_{n}$ obtemos uma outra sequência discreta $D_{n}$ da seguinte forma.

Se $R_{n} \geq 2,5 \% \Rightarrow D_{n}=1$

Se $1,5 \% \leq R_{n}<2,5 \% \Rightarrow D_{n}=2$

Se $0,5 \% \leq R_{n}<1,5 \% \Rightarrow D_{n}=3$

Se $-0,5 \% \leq R_{n}<0,5 \% \Rightarrow D_{n}=4$

Se $-1,5 \% \leq R_{n}<-0,5 \% \Rightarrow D_{n}=5$

Se $-2,5 \% \leq R_{n}<-1,5 \% \Rightarrow D_{n}=6$

Se $R_{n}<-2,5 \% \Rightarrow D_{n}=7$

A sequência a ser analisada é $\left\{D_{n}\right\}, \operatorname{com}\left\{D_{n}\right\}$ discreta e com espaço de estados $S=\{1,2,3,4,5,6,7\}$. Notando que $D_{n}$ tem dependência dos valores anteriores via $P_{n}$, desejamos saber qual o tamanho da dependência que essa sequência possui, ou seja, qual ordem melhor ajusta essa dependência. Utilizando os testes descritos na Seção 2 e calculando os estimadores, obtivemos as seguintes tabelas:

Tabela 1: Companhia aéria GOLL

\begin{tabular}{cccc}
\hline Ordem & AIC & BIC & EDC \\
\hline 0 & 402.14 & 404.47 & 395.43 \\
1 & 456.97 & 473.32 & 410.04 \\
2 & 863.61 & 978.02 & 535.08 \\
3 & 4217.02 & 5017.92 & 1917.35 \\
4 & 28825.24 & 34431.57 & 12727.62 \\
\hline
\end{tabular}

Tabela 3: Frigorífico JBS

\begin{tabular}{cccc}
\hline Ordem & AIC & BIC & EDC \\
\hline 0 & 422.19 & 424.52 & 415.48 \\
1 & 473.22 & 489.56 & 426.28 \\
2 & 847.16 & 961.58 & 518.64 \\
3 & 4195.49 & 4996.40 & 1895.83 \\
4 & 28830.06 & 34436.39 & 12732.43 \\
\hline
\end{tabular}

Tabela 2: Companhia de cartões CIELO

\begin{tabular}{cccc}
\hline Ordem & AIC & BIC & EDC \\
\hline 0 & 419.28 & 421.61 & 412.57 \\
1 & 468.99 & 485.34 & 422.06 \\
2 & 853.93 & 968.34 & 525.41 \\
3 & 4189.91 & 4990.82 & 1890.25 \\
4 & 28828.86 & 34435.18 & 12731.23 \\
\hline
\end{tabular}

\section{Conclusões}

Vimos neste trabalho que quando aplicado os estimadores de ordem para determinar a ordem de dependência entre os retornos diários das ações, os três estimadores utilizados AIC, BIC e EDC, nos mostraram que a ordem foi zero, ou seja, que não existe uma dependência entre os retornos atuais com os retornos passados e que a dinâmica dos preços seguem a proporção do comportamento passado. Motiva-nos, investigar, em trabalhos vindouros, o que acontece com essa dependência quando considerados retornos semanais e mensais, ao invés dos retornos diários tratados no presente trabalho. Adicionalmente, uma análise num conjunto de ações mais amplo, em intervalo de observação maior (ou menor), separada por setores, se fizesse necessária a fim de verificar se a ausência de dependência percebida neste trabalho é geral ou setorial. 


\section{Agradecimento}

Agradecemos ao Departamento de Matemática da UFRN que nos proporcionou a infra-estrutura para a realização deste trabalho, bem como aos pareceristas cujos apontamentos tornaram melhor nosso texto.

\section{Referências}

[1] Akaike,H. , A New Look at the Statistical Model Identification, IEEE Trans. Autom. Cont., 19, 716-723, 1974.

[2] Bhusal, M.K., Application of Markov Chain Model in the Stock Market Trend Analysis of Nepal , International Journal of Scientific \& Engineering Research Volume 8, Issue 10, October2017,ISSN 2229-5518.

[3] Cechin, R.B. e Corso,L.L. , High-order Multivatiate Markov Chain Applied in Dow Jones and Ibovespa Indexes, Pesquisa Operacional 39(1):205-223,2019. ISSN 0101-7438.

[4] Chiu, C.W.J., Harris R., Stoja, E. e Chin M., Financial market volatility, macroeconomic fundamentals and investor sentiment., Journal of Banking \& Finance, 92: 130-145,2018.

[5] Choji, D.N., Eduno, S.N., Kassem, G.T., Markov Chain Model Application on Share Price Movement in Stock Market: Journal of Computer Engineering and Intelligent Systems. www. iiste.org, Vol.4, No. 10; 2013.

[6] Csiszár,I. \& P. C. Shields, The Consistency of the BIC Markov Order Estimator, Ann. Statist., 28, no. 6, 1601-1619, 2000.

[7] Dorea,C. C. Y., Optimal Penalty Term for EDC Markov Chain Order Estimator, Annales de l'ISUP, 52, 15-26, 2008.

[8] Doubleday, K.J. e Esunge, J.N. , Application of Markov Chains to Stock Trends, Journal of Mathematics and Statistics 7 (2): 103-106, 2011 . ISSN 1549-3644.

[9] Isaacson, D.L. e Madsen,R.W. , 1985. Markov Chains: Theory and Applications. 1st Edn., R.E. Krieger Pub. Co., New York, ISBN-13: 9780898748345.

[10] Katz, R. W. ,On Some Criteria for Estimating the Order of Markov Chain, Technometrics, 23, 243-249, 1981.

[11] Mettle F.O., Boi,Q.E.N. , Laryea, R.A., A Methodology for Stochastic Analysis of Share Price of Markov Chains with Finite States: SpringerPlus, doi: 10.1186/2193-1801-3-657; 2014.

[12] Schwarz,G., Estimating the Dimension of a Model, Ann. Statist., 6, 461-464, 1978.

[13] Tong,H. , Determination of the Order of a Markov Chains by Akaike's Information Criterion, J. App. Probab., 12, 488-497, 1975.

[14] Zhang D., Zhang X., Study on Forecasting the Stock Market Trend Based on Stochastic Analysis Method: International Journal of Business and Management. Vol. 4, No. 6. June; 2009.

[15] Zhao,H. C. , Dorea,C. C. Y. e Gonçalves,C. R. , On Determination of the Order of a Markov Chains, Stat. Infer. for Stoc. Processes, 4, 273-282, 2001. 